\title{
Subset models for justification logic ${ }^{\star}$
}

\author{
Eveline Lehmann and Thomas Studer \\ Institute of Computer Science, University of Bern, Switzerland \\ \{lehmann, tstuder\}@inf .unibe.ch
}

\begin{abstract}
We introduce a new semantics for justification logic based on subset relations. Instead of using the established and more symbolic interpretation of justifications, we model justifications as sets of possible worlds. We introduce a new justification logic that is sound and complete with respect to our semantics. Moreover, we present another variant of our semantics that corresponds to traditional justification logic.

These types of models offer us a versatile tool to work with justifications, e.g. by extending them with a probability measure to capture uncertain justifications. Following this strategy we will show that they subsume Artemov's approach to aggregating probabilistic evidence.
\end{abstract}

Keywords: justification logic $\cdot$ semantics $\cdot$ probabilistic evidence

\section{Introduction}

Justification logic is a variant of modal logic that includes terms representing explicit evidence. A formula of the form $t: A$ means that $t$ justifies $A$ (or $t$ represents evidence for $A$, or $t$ is a proof of $A$ ). Justification logic has been introduced by Artemov [3,4] to give a classical provability interpretation to S4. Later it turned out that this approach is not only useful in proof theory $[4,15]$ but also in epistemic logic $[5,6,11,12]$. For a general overview on justification logic, we refer to $[8,2,16]$.

There are various kinds of semantics available for justification logic. Most of them interpret justification terms in a symbolic way. In provability interpretations [4,15], terms represent (codes of) proofs in formal system like Peano arithmetic. In Mkrtychev models [18], which are used to obtain decidability, terms are represented as sets of formulas. In Fitting models [13], the evidence relation maps pairs of terms and possible worlds to sets of formulas. In modular models $[7,14]$, the logical type a justification is a set of formulas, too. Notable exceptions are $[1,9]$ where terms are interpreted as sets of possible worlds. However, these papers do not consider the usual term structure of justification logics. Also note that there are topological approaches to evidence available $[10,21,22]$, which, however, do not feature justifications explicitly in their language.

\footnotetext{
* This work was supported by the Swiss National Science Foundation grant 200021_165549.
} 
It is the aim of this paper to provide a new semantics, called subset semantics for justification logic that interprets terms as sets of possible worlds and operations on terms as operations on sets of possible worlds. We will then say that $t: A$ is true if $A$ is true in all worlds belonging to the interpretation of $t$. We give a systematic study of this new semantics including soundness and completeness results and we show that the approach of [1] can be seen as a special case of our semantics.

Usually, justification logic includes an application operator that represents modus ponens (MP) on the level of terms. We provide two approaches to handle this operator in our semantics. The first is to include a new constant $c^{\star}$, which is interpreted as the set of all worlds closed under (MP) and then use this new constant to define an application operator. Unlike in traditional justification logic, this application operator will be commutative. The second way is to include a (non-commutative) application operator directly. However, this leads to some quite cumbersome definitions.

Another difference between our semantics and many other semantics for justification logic is that we allow non-normal (impossible) worlds. They are usually needed to model the fact that agents are not omniscient and that they do not see all consequences of the facts they are already aware of. In an impossible world both $A$ and $\neg A$ may be true or none of them. This way of using impossible worlds was investigated by Veikko Rantala $[19,20]$.

We start with presenting the $c^{\star}$-subset models with the corresponding syntax, axioms and semantics and proving soundness and completeness. In a second part we will present the alternative approach, i.e. keeping the $(\mathrm{j})$-axiom and dealing with some cumbersome definitions within the semantics. It will be shown that the corresponding models are sound and complete as well. In a last section we will show that $c^{\star}$-subset models can be used to reason about uncertain knowledge by referring to Artemov's work on aggregating probabilistic evidence.

\section{$2 \quad L_{\text {CS }}^{\star}$-subset models}

\subsection{Syntax}

Justification terms are built from countably many constants $c_{i}$ and variables $x_{i}$ and the special and unique constant $c^{\star}$ according to the following grammar:

$$
t::=c_{i}\left|x_{i}\right| c^{\star}|(t+t)| ! t
$$

The set of terms is denoted by Tm. The operation + is left-associative.

Formulas are built from countably many atomic propositions $p_{i}$ and the symbol $\perp$ according to the following grammar:

$$
F::=p_{i}|\perp| F \rightarrow F \mid t: F
$$

The set of atomic propositions is denoted by Prop and the set of all formulas is denoted by $\mathcal{L}_{J}$. The other classical Boolean connectives $\neg, \top, \wedge, \vee, \leftrightarrow$ are defined as usual. 
We investigate a family of justification logics that differ in their axioms and how the axioms are justified. We have two sets of axioms, the first axioms are:

cl all axioms of classical propositional logic;

j+ $s: A \vee t: A \rightarrow(s+t): A$

$\mathbf{j} \mathbf{c}^{\star} \mathrm{c}^{\star}: A \wedge \mathrm{c}^{\star}:(A \rightarrow B) \rightarrow \mathrm{c}^{\star}: B$.

The set of these axioms is denoted by $L_{\alpha}^{\star}$.

There is another set of axioms:

$\mathbf{j} 4 t: A \rightarrow ! t:(t: A)$;

jd $t: \perp \rightarrow \perp$;

jt $t: A \rightarrow A$.

This set is denoted by $L_{\beta}^{\star}$. It is easy to see that $\mathbf{j d}$ is a special case of $\mathbf{j t}$. By $\mathbf{L}^{\star}$ we denote all logics that are composed from the whole set $L_{\alpha}^{\star}$ and some subset of $L_{\beta}^{\star}$. Moreover, a justification logic $L^{\star}$ is defined by the set of axioms and its constant specification CS that determines which constant justifies which axiom. So the constant specification is a set

$$
\mathrm{CS} \subseteq\left\{(c, A) \mid c \text { is a constant and } A \text { is an axiom of } \mathrm{L}^{\star}\right\}
$$

In this sense $L_{C S}^{\star}$ denotes the logic $L^{\star}$ with the constant specification CS. To deduce formulas in $L_{C S}^{\star}$ we use a Hilbert system given by $L^{\star}$ and the rules modus ponens:

$$
\frac{A \quad A \rightarrow B}{B}(\mathrm{MP})
$$

and axiom necessitation

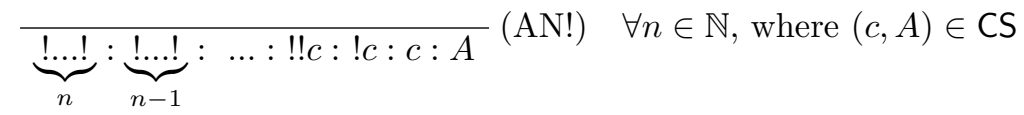

\subsection{Semantics}

Definition 1 ( $L_{C S}^{\star}$-subset models) Given some logic $L^{\star}$ and some constant specification $\mathrm{CS}$, then an $\mathrm{L}_{\mathrm{CS}}^{\star}$-subset model $\mathcal{M}=\left(W, W_{0}, V, E\right)$ is defined by:

- $W$ is a set of objects called worlds.

$-W_{0} \subseteq W$ and $W_{0} \neq \emptyset$.

- $V: W \times \mathcal{L}_{J} \rightarrow\{0,1\}$ such that for all $\omega \in W_{0}, t \in \mathrm{Tm}, F, G \in \mathcal{L}_{J}$ :

- $V(\omega, \perp)=0$;

- $V(\omega, F \rightarrow G)=1 \quad$ iff $\quad V(\omega, F)=0$ or $V(\omega, G)=1$;

- $V(\omega, t: F)=1 \quad$ iff $E(\omega, t) \subseteq\{v \in W \mid V(v, F)=1\}$.

- $E: W \times \operatorname{Tm} \rightarrow \mathcal{P}(W)$ that meets the following conditions where we use

$$
[A]:=\{\omega \in W \mid V(\omega, A)=1\} .
$$

For all $\omega \in W_{0}$, and for all $s, t \in \mathrm{Tm}$ : 
- $E(\omega, s+t) \subseteq E(\omega, s) \cap E(\omega, t)$;

- $E\left(\omega, c^{\star}\right) \subseteq W_{M P}$ where $W_{M P}$ is the set of deductively closed worlds, see below;

- if $\boldsymbol{j} \boldsymbol{d} \in \mathrm{L}^{\star}$, then $\exists v \in W_{0}$ with $v \in E(\omega, t)$;

- if $\boldsymbol{j} \boldsymbol{t} \in \mathrm{L}^{\star}$, then $\omega \in E(\omega, t)$;

- if $j 4 \in \mathrm{L}^{\star}$, then

$$
\begin{aligned}
E(\omega, ! t) \subseteq & \left\{v \in W \mid \forall F \in \mathcal{L}_{J}(V(\omega, t: F)=1 \Rightarrow V(v, t: F)=1)\right\} ;
\end{aligned}
$$

- for all $n \in \mathbb{N}$ and for all $(c, A) \in \mathrm{CS}: E(\omega, c) \subseteq[A]$ and

$$
E(\omega, \underbrace{! \ldots !}_{n} c) \subseteq[\underbrace{! \ldots !}_{n-1} c: \ldots . . ! c: c: A] .
$$

The set $W_{M P}$ is formally defined as follows:

$$
\begin{gathered}
W_{M P}:=\left\{\omega \in W \mid \forall A, B \in \mathcal{L}_{J}((V(\omega, A)=1 \text { and } V(\omega, A \rightarrow B)=1)\right. \\
\text { implies } V(\omega, B)=1)\} .
\end{gathered}
$$

So $W_{M P}$ collects all the worlds where the valuation function is closed under modus ponens. $W_{0}$ is the set of normal worlds. The set $W \backslash W_{0}$ consists of the non-normal worlds. Moreover, using the notation introduced by (1), we can read the condition on $V$ for justification terms $t: F$ as:

$$
V(\omega, t: F)=1 \quad \text { iff } \quad E(\omega, t) \subseteq[F]
$$

Since the valuation function $V$ is defined on worlds and formulas, the definition of truth is pretty simple:

Definition 2 (Truth in $\mathrm{L}_{\mathrm{CS}}^{\star}$-subset models) Let $\mathcal{M}=\left(W, W_{0}, V, E\right)$ be an $\mathrm{L}_{\mathrm{CS}}^{\star}$-subset model, $\omega \in W$ and $F \in \mathcal{L}_{J}$. We define the relation $\Vdash$ as follows:

$$
\mathcal{M}, \omega \Vdash F \quad \text { iff } \quad V(\omega, F)=1
$$

\subsection{Soundness}

Since non-normal worlds will not be sound even with respect to the axioms of classical logic, we only have soundness within $W_{0}$.

Theorem 3 (Soundness of $\mathrm{L}_{\mathrm{CS}}^{\star}$-subset models) For any justification logic $\mathrm{L}_{\mathrm{CS}}^{\star}$ and any formula $F \in \mathcal{L}_{J}$ :

$$
\mathrm{L}_{\mathrm{CS}}^{\star} \vdash F \quad \Rightarrow \quad \mathcal{M}, \omega \Vdash F \quad \text { for all } \mathrm{L}_{\mathrm{CS}}^{\star} \text {-subset models } \mathcal{M} \text { and all } \omega \in W_{0}
$$

The proof straight forward is by induction on the length on the derivation of $F$ and can be found in [17].

The $\mathbf{j}$-axiom $s:(A \rightarrow B) \rightarrow(t: A \rightarrow s \cdot t: B)$ is not part of our logic. Using the $\left(\mathrm{c}^{\star}\right)$-axiom, we can define an application operation such that the $\mathbf{j}$-axiom is valid. 
Definition 4 (Application) We introduce a new abbreviation - on terms by:

$$
s \cdot t:=s+t+c^{\star}
$$

Lemma 5 (The "j-axiom" follows) For all $\mathcal{M}=\left(W, W_{0}, V, E\right), \omega \in W_{0}$, $A, B \in \mathcal{L}_{J}$ and $s, t \in \mathrm{Tm}:$

$$
\mathcal{M}, \omega \Vdash s:(A \rightarrow B) \rightarrow(t: A \rightarrow s \cdot t: B)
$$

The proof is straight forward and can be found in appendix A.

Lemma 6 (Application is communative) Let $\mathcal{M}=\left(W, W_{0}, V, E\right)$ be an $\mathrm{L}^{\star}$ subset model, $\omega \in W, A \in \mathcal{L}_{J}, s, t \in \mathrm{Tm}$, then:

$$
\mathcal{M}, \omega \Vdash s \cdot t: A \leftrightarrow t \cdot s: A
$$

The proof is straight forward by using the commutativity of intersection. For details see [17].

So it does not make a difference, whether we first have evidence for $B \rightarrow A$ and then for $B$ or vice versa. In other words, the order in which evidence is presented does not matter.

\subsection{Completeness}

To prove completeness we will construct a canonical model and then show that for every formula $F$ that is not derivable in $\mathrm{L}_{\mathrm{CS}}^{\star}$, there is a model $\mathcal{M}^{C}$ with a world $\Gamma \in W_{0}^{C}$ s.t. $\mathcal{M}^{C}, \Gamma \Vdash \neg F$. Before we start with the definition of the canonical model, we must do some preliminary work. We will first prove that our logics are conservative extensions of classical logic. With this result we can argue, that the empty set is consistent and hence can be extended to so-called maximal $L_{C S}^{\star}$-consistent sets of formulas. These sets will be used to build the $W_{0}$-worlds in the canonical model.

Theorem 7 (Conservativity) All logics $L^{\star}$ presented are conservative extensions of the classical logic $\mathrm{CL}$, i.e. for any formula $F \in \mathrm{L}_{\mathrm{cp}}$ :

$$
\mathrm{L}^{\star} \vdash F \quad \Leftrightarrow \quad \mathrm{CL} \vdash F
$$

The proof is standard and can be found in [17].

Definition 8 (Consistency) A logical theory $\mathrm{L}$ is called consistent, if $\mathrm{L} \forall \perp$. A set of formulas $\Gamma \subset \mathcal{L}_{J}$ is called $\mathrm{L}$-consistent if $\mathrm{L} \forall \forall \wedge \Sigma \rightarrow \perp$ for every finite $\Sigma \subseteq \Gamma$. A set of formulas $\Gamma$ is called maximal $\mathbf{L}$-consistent, if it is $\mathbf{L}$-consistent and none of its proper supersets is.

Since all presented logics are conservative extensions of $\mathrm{CL}$, we have the following consistency result.

Lemma 9 (Consistency of the logics) All presented logics are consistent. 
As usual, we have a Lindenbaum lemma and the usual properties of maximal consistent sets hold, see, e.g., [16].

Lemma 10 (Lindenbaum Lemma) Given some logic $\mathrm{L}$, then for each $\mathrm{L}$ - consistent set of formulas $\Gamma \subset \mathcal{L}_{J}$ there exists a maximal consistent set $\Gamma^{\prime}$ such that $\Gamma \subseteq \Gamma^{\prime}$.

Lemma 11 (Properties of maximal consistent sets) Given some logic $\mathrm{L}$ and its language $\mathcal{L}_{J}$. If $\Gamma$ is a maximal $\mathrm{L}$-consistent set, then for all $F, G \in \mathcal{L}_{J}$ :

(1) if $\mathrm{L} \vdash F$, then $F \in \Gamma$;

(2) $F \in \Gamma$ if and only if $\neg F \notin \Gamma$;

(3) $F \rightarrow G \in \Gamma$ if and only if $F \notin \Gamma$ or $G \in \Gamma$;

(4) $F \in \Gamma$ and $F \rightarrow G \in \Gamma$ imply $G \in \Gamma$.

Definition 12 (Canonical Model) For a given logic $\mathrm{L}_{\mathrm{CS}}^{\star}$ we define the canonical model $\mathcal{M}^{C}=\left(W^{C}, W_{0}^{C}, V^{C}, E^{C}\right)$ by:

$-W^{C}=\mathcal{P}\left(\mathcal{L}_{J}\right)$.

- $W_{0}^{C}=\left\{\Gamma \in W^{C} \mid \Gamma\right.$ is maximal $\mathrm{L}_{\mathrm{CS}}^{\star}-$ consistent set of formulas $\}$.

$-V^{C}: V^{C}(\Gamma, F)=1 \quad$ iff $\quad F \in \Gamma$

- $E^{C}:$ With $\Gamma / t:=\left\{F \in \mathcal{L}_{J} \mid t: F \in \Gamma\right\}$ and

$W_{M P}^{C}:=\left\{\Gamma \in W^{C} \mid \forall A, B \in \mathcal{L}_{J}:\right.$ if $A \rightarrow B \in \Gamma$ and $A \in \Gamma$ then $\left.B \in \Gamma\right\}$

we define :

$$
\begin{aligned}
E^{C}(\Gamma, t) & =\left\{\Delta \in W^{C} \mid \Delta \supseteq \Gamma / t\right\} \text { for } t \neq \mathrm{c}^{\star} ; \\
E^{C}\left(\Gamma, \mathrm{c}^{\star}\right) & =\left\{\Delta \in W_{M P}^{C} \mid \Delta \supseteq \Gamma / \mathrm{c}^{\star}\right\} .
\end{aligned}
$$

Now we must show that the canonical model is indeed an $\mathrm{L}_{\mathrm{CS}}^{\star}$-subset model.

Lemma 13 The canonical model $\mathcal{M}^{C}$ is an $\mathrm{L}_{\mathrm{CS}}^{\star}$-subset model.

The proof can be found in appendix B. The Truth Lemma follows very closely:

Lemma 14 (Truth Lemma) Let $\mathcal{M}^{C}=\left(W^{C}, W_{0}^{C}, E^{C}, V^{C}\right)$ be a canonical model, then for any $\Gamma \in W_{0}^{C}$ :

$$
\mathcal{M}^{C}, \Gamma \Vdash F \text { if and only if } F \in \Gamma \text {. }
$$

Proof.

$$
\mathcal{M}^{C}, \Gamma \Vdash F \stackrel{\text { Def. } 2}{\Longleftrightarrow} V^{C}(\Gamma, F)=1 \stackrel{\text { Def. } 12}{\Longleftrightarrow} F \in \Gamma .
$$

Hence each maximal $L_{C S}^{\star}$-consistent set is represented by some world in the canonical model and thus completeness follows directly:

Theorem 15 (Completeness) Given some logic $\mathrm{L}_{\mathrm{CS}}^{\star}$, then

$$
\mathcal{M}, \Gamma \Vdash F \quad \text { for all } \mathrm{L}_{\mathrm{CS}}^{\star} \text {-subset models } \mathcal{M} \text { and for all } \Gamma \in W_{0} \Longrightarrow \mathrm{L}_{\mathrm{CS}}^{\star} \vdash F \text {. }
$$

Proof. The proof works with contraposition: Assume that $\mathrm{L}_{\mathrm{CS}}^{\star} \forall \forall$. Then $\{\neg F\}$ is $L_{C S}^{\star}$-consistent and by the Lindenbaum Lemma contained in some maximal $\mathrm{L}_{\mathrm{CS}}^{\star}$-consistent world $\Gamma$ of the canonical model $\mathcal{M}^{C}$. Then $\mathcal{M}^{C}, \Gamma \Vdash F$. 


\section{$3 \quad \mathrm{~L}_{\mathrm{CS}}^{\mathrm{A}}$-subset models}

In this part we present an alternative definition of subset models for justification logic that directly interprets the application operator. Hence we work with the standard language of justification logic and we consider the $\mathbf{j}$-axiom instead of the axiom $\left(c^{\star}\right)$.

\subsection{Syntax}

In this section, justification terms are built from constants $c_{i}$ and variables $x_{i}$ according to the following grammar:

$$
t::=c_{i}\left|x_{i}\right|(t \cdot t)|(t+t)| ! t
$$

This set of terms is denoted by $\operatorname{Tm}^{\mathrm{A}}$. The operations $\cdot$ and + are left-associative and ! binds stronger than anything else. Formulas are built from atomic propositions $p_{i}$ and the following grammar:

$$
F::=p_{i}|\perp| F \rightarrow F \mid t: F
$$

The set of atomic propositions is denoted by Prop and the set of all formulas is denoted by $\mathcal{L}_{J}^{\mathrm{A}}$. Again we use the other logical connectives as abbreviations.

As in the first section, we investigate again a whole family of logics. They are arranged in two sets of axioms. The first set, denoted by $L_{\alpha}^{A}$ contains the following axioms:

cl all axioms of classical propositional logic;

j $s:(A \rightarrow B) \rightarrow(t: A \rightarrow s \cdot t: B)$;

$\mathbf{j}+s: A \vee t: A \rightarrow(s+t): A$.

The other is identical to $L_{\beta}^{\star}$ (modulo the different language) and contains:

$\mathbf{j} 4 t: A \rightarrow ! t:(t: A)$;

jd $t: \perp \rightarrow \perp$;

jt $t: A \rightarrow A$.

For the sake of uniformity we denote this set of axioms by $L_{\beta}^{A}$. By $L^{A}$ we denote all logics that are composed from the whole set $L_{\alpha}^{A}$ and some subset of $L_{\beta}^{A}$.

There are no differences between these logics and the ones of the former section except in case of application. Therefore we skip all the details already mentioned and proved before.

CS and $L_{C S}^{A}$ are defined as before except that the corresponding logic has changed as mentioned. And deducing formulas in $\mathrm{L}_{\mathrm{CS}}^{\mathrm{A}}$ works the same as in the previous section. 


\subsection{Semantics}

Definition 16 ( $\mathrm{L}_{C S}^{\mathrm{A}}$-subset models) Given some logic $\mathrm{L}_{C S}^{\mathrm{A}}$ then an $\mathrm{L}_{\mathrm{CS}}^{\mathrm{A}}$-subset model $\mathcal{M}=\left(W, W_{0}, V, E\right)$ is defined like an $\mathrm{L}_{\mathrm{CS}}^{\star}$-subset model where

$$
E: W \times \operatorname{Tm}^{\mathrm{A}} \rightarrow \mathcal{P}(W)
$$

meets the following condition for terms of the form $s \cdot t$ :

$$
E(\omega, s \cdot t) \subseteq\left\{v \in W \mid \forall F \in \operatorname{APP}_{\omega}(s, t)(v \in[F])\right\},
$$

where we use

$\operatorname{APP}_{\omega}(s, t):=\left\{F \in \mathcal{L}_{J}^{\mathrm{A}} \mid \exists H \in \mathcal{L}_{J}^{\mathrm{A}}\right.$ s.t. $E(\omega, s) \subseteq[H \rightarrow F]$ and $\left.E(\omega, t) \subseteq[H]\right\}$.

The set $\operatorname{APP}_{\omega}(s, t)$ contains all formulas that are colloquially said derivable by applying modus ponens to a formula justified by $s$ and a formula justified by $t$.

Truth in an $\mathrm{L}_{\mathrm{CS}}^{\mathrm{A}}$-subset models is defined as before.

Definition 17 (Truth in $\mathrm{L}_{C S}^{\mathrm{A}}$-subset models) For an $\mathrm{L}_{C S}^{\mathrm{A}}$-subset model $\mathcal{M}=$ $\left(W, W_{0}, V, E\right)$ and a world $\omega \in W$ and a formula $F$ we define the relation $\Vdash$ as follows:

$$
\mathcal{M}, \omega \Vdash F \quad \text { iff } \quad V(\omega, F)=1 .
$$

\subsection{Soundness}

Theorem 18 (Soundness of $\mathrm{L}_{\mathrm{CS}}^{\mathrm{A}}$-subset models) For any justification logic $\mathrm{L}^{\mathrm{A}}$, any constant specification $\mathrm{CS}$ and any formula $\mathrm{F}$ :

$$
\mathrm{L}_{\mathrm{CS}}^{\mathrm{A}} \vdash F \Rightarrow \mathcal{M}, \omega \Vdash F \quad \text { for all } \mathrm{L}_{\mathrm{CS}}^{\mathrm{A}}-\text { subset models } \mathcal{M} \text { and all } \omega \in W_{0} \text {. }
$$

The proof is straight forward by induction on the length of the derivation and can be found in [17].

\subsection{Completeness}

Before we start defining a canonical model, we have to do the same preliminary work for $L_{C S}^{A}$ as we had to do in the previous section for $L_{C S}^{\star}$. Since the logics $L_{C S}^{\star}$ from the former section differ only in one axiom, i.e. $\mathbf{j}$ replaces $\mathbf{j} \mathbf{c}^{\star}$, we skip all the parts that are already done and focus on the changes that it brings about.

As before, we have a conservativity and consistency result.

Theorem 19 (Conservativity) All logics $\mathrm{L}^{\mathrm{A}}$ presented are conservative extensions of the classical logic $\mathrm{CL}$, i.e. for any formula $F \in \mathrm{L}_{\mathrm{cp}}$ :

$$
\mathrm{L}^{\mathrm{A}} \vdash F \quad \Leftrightarrow \quad \mathrm{CL} \vdash F .
$$

Lemma 20 (Consistency of $\mathrm{L}^{\mathrm{A}}$ ) All logics in $\mathrm{L}^{\mathrm{A}}$ are consistent. 
All the other ingredients we needed in the former section to define and further develop the canonical model were generally defined and proven and can be adopted without additional effort.

To prove completeness we define a canonical model as follows:

Definition 21 (Canonical Model) For a given logic $\mathrm{L}^{\mathrm{A}}$ and a constant specification CS we define the canonical model $\mathcal{M}^{C}=\left(W^{C}, W_{0}^{C}, V^{C}, E^{C}\right)$ by:

$-W^{C}=\mathcal{P}\left(\mathcal{L}_{J}^{\mathrm{A}}\right)$

- $W_{0}^{C}=\left\{\Gamma \in W^{C} \mid \Gamma\right.$ is maximal $\mathrm{L}_{\mathrm{CS}}^{\mathrm{A}}$ - consistent set of formulas $\}$;

$-V^{C}: V^{C}(\Gamma, F)=1 \quad$ iff $\quad F \in \Gamma$;

- $E^{C}: E^{C}(\Gamma, t)=\{\Delta \in W \mid \Delta \supseteq \Gamma / t\}$.

Now we must show that such a canonical model is in fact a subset model.

Lemma 22 The canonical model $\mathcal{M}^{C}$ is an $\mathrm{L}_{\mathrm{CS}}^{\mathrm{A}}$-subset model.

The proof is very similar to the proof of Lemma 13 and can be found in appendix C

Lemma 23 (Truth Lemma) Let $\mathcal{M}^{C}=\left(W^{C}, W_{0}^{C}, E^{C}, V^{C}\right)$ be some canonical $\mathrm{L}_{\mathrm{CS}}^{\mathrm{A}}$-subset model, then for all $\Gamma \in W_{0}$ :

$$
\mathcal{M}^{C}, \Gamma \Vdash F \text { if and only if } F \in \Gamma \text {. }
$$

Proof.

$$
\mathcal{M}^{C}, \Gamma \Vdash F \stackrel{\text { Def. } 17}{\Longleftrightarrow} V^{C}(\Gamma, F)=1 \stackrel{\text { Def. } 21}{\Longleftrightarrow} F \in \Gamma .
$$

Theorem 24 (Completeness) Given some constant specification CS then

$$
\mathcal{M}, \Gamma \Vdash F \text { for all models } \mathcal{M} \text { and for all } \Gamma \in W_{0} \Longrightarrow \mathrm{L}_{\mathrm{CS}}^{\mathrm{A}} \vdash F \text {. }
$$

Proof. The proof is analogue to the one of Theorem 15.

\section{Artemov's aggregated evidence and $L_{c S}^{\star}$-subset models}

Artemov [1] considers the case in which we have a database, i.e. a set of propositions $\Gamma=\left\{F_{1}, \ldots F_{n}\right\}$ with some kind of probability estimates and in which we also have some proposition $X$ that logically follows from $\Gamma$. Then we can search for the best justified lower bound for the probability of $X$. He presents us a nice way to find this lower bound. To find it, he assumes probability events $u_{1}, \ldots, u_{n}$, each of them supporting some proposition in $\Gamma$, i.e. $u_{i}: F_{i}$, and calculates some aggregated evidence $e\left(u_{1}, \ldots, u_{n}\right)$ for $X$ with them. The probability of $e$ then provides a tight lower bound for the probability of $X$.

The trick he uses is the following:

(1) First he collects all subsets $\Delta_{i}$ of $\Gamma$ which support $X$, i.e. $\Delta_{i} \vdash X$, and creates a new evidence $t_{i}$ from all the corresponding $u_{i_{j}}$ s.t. $u_{i_{j}}: F_{i_{j}}$ for each $F_{i_{j}} \in \Delta_{i}$. 
(2) In the second step he combines all these new pieces of evidence to a new evidence (the so-called aggregated evidence) that actually is the greatest evidence supporting $X$.

The model he has in mind contains some evaluation in a probability space $(\Omega, \mathcal{F}, P)$ with a mapping $\star$ from propositions to $\Omega$ and evidence terms to $\mathcal{F}$ that meets some restrictions (for more details on this see [1]). Step (1) is to create a new evidence $t_{i}$ for each $\Delta_{i}$ described above, which consists of the intersection of the corresponding $u_{i_{j}}$ s.

$$
t_{i}:=\bigcap\left\{u_{i_{j}} \mid u_{i_{j}} \subseteq F_{i_{j}}^{\star} \text { for some } F_{i_{j}} \in \Delta_{i}\right\} .
$$

Step (2) then is to union all these pieces of evidence to a new so-called aggregated evidence:

$$
\operatorname{AE}^{\Gamma}(\mathrm{X}):=\bigcup\left\{t_{i} \mid t_{i} \text { is an evidence for } X \text { obtained by step (1) }\right\} .
$$

On the syntactic side evidence terms are built from variables $u_{1}, \ldots, u_{n}$, constants 0 and 1 and operations $\cap$ and $\cup$, where st is used as an abbreviation for $s \cap t$. With this we can built a free distributive lattice $\mathcal{L}_{n}$ where $s t$ is the meet and $s \cup t$ is the join of $s$ and $t, 0$ is the bottom and 1 the top element of this lattice. Moreover Artemov defines formulas in a usual way from propositional letters $p, q, r, \ldots$ by the usual connectives and adds formulas of the kind $t: F$ where $t$ is an evidence term and $F$ a purely propositional formula.

The logical postulates of the logic of Probabilistic Evidence PE are:

(1) axioms and rules of classical logic in the language of PE;

(2) $s:(A \rightarrow B) \rightarrow(t: A \rightarrow[s t]: B)$;

(3) $(s: A \wedge t: A) \rightarrow[s \cup t]: A$;

(4) $1: A$, where $A$ is a propositional tautology,

$0: F$, where $F$ is a propositional formula;

(5) $t: X \rightarrow s: X$, for any evidence terms $s$ and $t$ such that $s \preceq t$ in $\mathcal{L}_{n}$.

Artemov presents Soundness and Completeness proofs connecting PE with the presented semantic, for more details see [1].

Before we can start adapting Artemovs approach to our models, we have to point out some differences between the semantics and syntax used. First, contrary to the models of Artemov, subset models may contain inconsistent worlds, but this does not significantly affect the applicability of Artemov's approach on them.

Another difference is that our evidence function has another domain. In Artemov's models the evidence functions is $E: \operatorname{Tm} \rightarrow \mathcal{P}(\Omega)$ while in our models it is $E: W \times \mathrm{Tm} \rightarrow \mathcal{P}(W)$. This difference is due to the fact that we allow terms to justify non-purely propositional formulas. Although we need to adapt Artemov's definitions, these adaptations will maintain the essential characteristics. So let's adapt the $L_{C S}^{\star}$-subset models to aggregated $L_{C S}^{\star}$-subset models by first describing the new syntax for the terms: 
Definition 25 (Justification Terms) Justification terms are built from constants $0,1, c_{i}$ and variables $x_{i}$ and the special and unique constant $\mathrm{c}^{\star}$ according to the following grammar:

$$
t::=0|1| c_{i}\left|x_{i}\right| \mathrm{c}^{\star}|(t+t)|(t \cup t) \mid ! t
$$

This set of terms is denoted by $\mathrm{Tm}^{\mathrm{P}}$. As before, we introduce the abbreviation $s t:=s+t+\mathrm{c}^{\star}$.

Even though we have other operators as well, we can construct a free distributive lattice where we take $s+t$ as the meet of $s$ and $t, s \cup t$ as the join of them, 0 as the bottom element of the lattice. Note, that $s t$ then is the meet of $s, t$, and $\mathrm{c}^{\star}$. Moreover, 1 and $! t$ are treated like constants. ${ }^{1}$ As usual, we have

$$
s \preceq t \quad \text { iff } \quad s \cup t=t
$$
far.

So not all pairs of terms are comparable. But that has no consequences so

There is no difference to our subset models regarding the rules for forming formulas except that the terms are contained in $\mathrm{Tm}^{\mathrm{P}}$, of course. The set of formulas built according to these grammar and rules is denoted by $L_{\text {prob }}$.

In the definition of $\mathrm{L}_{\mathrm{CS}}^{\star}$-subset models we only change the conditions on the evidence function and the domain of $V$.

Definition 26 (PE-adapted subset models) An $\mathrm{L}_{\mathrm{CS}}^{\star}$ - subset model is called a $\mathrm{PE}$-adapted $\mathrm{L}_{\mathrm{CS}}^{\star}$-subset model if the valuation function and the evidence function meet the additional conditions respectively are redefined as follows:

$-V: W \times \mathrm{L}_{\text {prob }} \rightarrow\{0,1\}$ where all conditions listed in Definition 1 remain the same.

- For all $\omega \in W_{0}$ and for all $s, t \in \mathrm{Tm}^{\mathrm{P}}$ :

- $E(\omega, 1)=W_{0}$;

- $E(\omega, 0)=\emptyset$;

- $E(\omega, s \cup t)=E(\omega, s) \cup E(\omega, t)$.

And in fact, such an PE-adapted $\mathrm{L}_{\mathrm{CS}}^{\star}$-subset model is a model of probabilistic evidence PE.

Theorem 27 (Soundness) PE-adapted $\mathrm{L}_{\mathrm{CS}}^{\star}$-subset models $\mathcal{M}$ are sound with respect to probabilistic evidence $\mathrm{PE}$, i.e. for all $F \in \mathrm{L}_{\text {prob }}$

$\mathrm{PE} \vdash F \Rightarrow \mathcal{M}, \omega \Vdash F \quad$ for all $\mathrm{PE}$-adapted $\mathrm{L}_{\mathrm{CS}}^{\star}$-subset models and all $\omega \in W_{0}$.

The proof is by induction on the length of the derivation of $F$ and can be found in appendix D

\footnotetext{
${ }^{1}$ We do not claim that 1 is the top element since some set $E(\omega, t)$ for a world $\omega \in W_{0}$ and $t \in \mathrm{Tm}^{\mathrm{P}}$ may contain non-normal worlds. If we claimed that 1 was the top element we would obtain $t \preceq 1$ and furthermore the set $E(\omega, 1)$ would contain nonnormal worlds as well. But since in non-normal worlds axioms may not be true, $E(\omega, 1) \nsubseteq[A]$ for some axiom $A$ may be the case and therefore axiom (4) would fail.
} 
Theorem 28 (model existence) There exists a PE-adapted $\mathrm{L}_{C \mathrm{CS}}^{\star}$-subset model.

Proof. We construct a model $\mathcal{M}=\left\{W, W_{0}, V, E\right\}$ as follows:

$-W=W_{0}=\{\omega\}$.

- The valuation function is built bottom up:

(1) $V(\omega, \perp)=0$;

(2) $V(\omega, P)=1$, for all $P \in$ Prop;

(3) $V(\omega, A \rightarrow B)=1$ iff $V(\omega, A)=0$ or $V(\omega, B)=1$;

(4) $V(\omega, t: F)=1$ iff $t \geq 1$ or if $t \geq 1$ and $V(\omega, F)=1$.

$-E(\omega, t)= \begin{cases}\{\omega\} & \text { if } t \geq 1 \\ \emptyset & \text { otherwise. }\end{cases}$

It is straightforward to show that $\mathcal{M}$ is indeed a PE-adapted $\mathrm{L}_{\mathrm{CS}}^{\star}$-subset model. Let us only show the condition $E(\omega, s \cup t)=E(\omega, s) \cup E(\omega, t)$.

Suppose first $s, t \geq 1$, Then $E(\omega, s \cup t)=\emptyset=E(\omega, s)=E(\omega, t)$ and hence the claim follows immediately.

Suppose at least one term of $s$ and $t$ is in greater than 1, then $E(\omega, s)=\{\omega\}$ or $E(\omega, t)=\{\omega\}$ and hence $E(\omega, s) \cup E(\omega, t)=\{\omega\}$ and since $s \leq s \cup t$ and $t \leq s \cup t$ we obtain $s \cup t \geq 1$ and therefore $E(\omega, s \cup t)=\{\omega\}$, so the claim holds.

Note that we cannot use the canonical model to show that adapted subset models exists since in the canonical model

$$
E(\Gamma, s \cup t) \nsubseteq E(\Gamma, s) \cup E(\Gamma, t) .
$$

However, in an adapted model we need these sets to be equal (see Definition 26) since otherwise axioms (3) and (5) would not be sound.

\section{Conclusion}

We introduced a new semantics, called subset semantics, for justifications. So far, often a symbolic approach was used to interpret justifications. In our semantics, justifications are modeled as sets of possible worlds. We also presented a new justification logic that is sound and complete with respect to our semantics. Moroever, we studied a variant of subset models that corresponds to traditional justification logic.

Subset models provide a versatile tool to work with justifications. In particular, we can naturally extend them with probability measures to capture uncertain justifications. In the last part of the paper, we showed that subset models subsume Artemov's approach to aggregating probabilistic evidence. 


\section{A The "j-axiom" follows (Lemma 5)}

For all $\mathcal{M}=\left(W, W_{0}, V, E\right), \omega \in W_{0}, A, B \in \mathcal{L}_{J}$ and $s, t \in \mathrm{Tm}$ :

$$
\mathcal{M}, \omega \Vdash s:(A \rightarrow B) \rightarrow(t: A \rightarrow s \cdot t: B)
$$

Proof. Suppose $\mathcal{M}, \omega \Vdash s:(A \rightarrow B)$ and $\mathcal{M}, \omega \Vdash t: A$. Thus $E(\omega, s) \subseteq[A \rightarrow B]$ and $E(\omega, t) \subseteq[A]$. We find

$$
\begin{aligned}
& E(\omega, s \cdot t)=E\left(\omega, s+t+c^{\star}\right)= \\
& E(\omega, s) \cap E(\omega, t) \cap E\left(\omega, c^{\star}\right) \subseteq[A \rightarrow B] \cap[A] \cap E\left(\omega, c^{\star}\right) .
\end{aligned}
$$

Hence for all $v \in E(\omega, s \cdot t)$ we have $V(v, A \rightarrow B)=1$ and $V(v, A)=1$ and $v \in E\left(\omega, c^{\star}\right)$ and therefore $V(v, B)=1$. Hence $E(\omega, s \cdot t) \subseteq[B]$ and we obtain $\mathcal{M}, \omega \Vdash s \cdot t: B$.

\section{B The canonical model $\mathcal{M}^{C}$ defined in Definition 12 is an $L_{\mathrm{CS}}^{\star}$-subset model. (Lemma 13)}

Proof. In order to prove this, we have to show that $\mathcal{M}^{C}$ meets all the conditions we made for the valuation and evidence function and the constant specification i.e.:

(1) $W_{0}^{C} \neq \emptyset$.

(2) For all $\Gamma \in W_{0}^{C}$ :

(a) $V^{C}(\Gamma, \perp)=0$

(b) $V^{C}(\Gamma, F \rightarrow G)=1 \quad$ iff $\quad V^{C}(\Gamma, F)=0$ or $V^{C}(\Gamma, G)=1$;

(c) $V^{C}(\Gamma, t: F)=1 \quad$ iff $\quad E(\Gamma, t) \subseteq[F]$.

(3) For all $\Gamma \in W_{0}^{C}, F \in \mathcal{L}_{J}, s, t \in \mathrm{Tm}$ :

(a) $E^{C}(\Gamma, s+t) \subseteq E^{C}(\Gamma, s) \cap E^{C}(\Gamma, t)$;

(b) $E^{C}\left(\Gamma, \mathrm{c}^{\star}\right) \subseteq \bar{W}_{M P}^{C}$;

(c) if jd in $\mathrm{L}^{\star}: \forall \Gamma \in W_{0}^{C}$ and $\forall t \in \mathrm{Tm}: \exists v \in W_{0}^{C}$ s.t. $v \in E^{C}(\Gamma, t)$;

(d) if jt in $\mathrm{L}^{\star}: \forall \Gamma \in W_{0}^{C}$ and $\forall t \in \mathrm{Tm}: \Gamma \in E^{C}(\Gamma, t)$;

(e) if $\mathbf{j} 4$ in $L^{\star}$ :

$$
\begin{aligned}
& E^{C}(\Gamma, ! t) \subseteq \\
& \quad\left\{\Delta \in W^{C} \mid \forall F \in \mathcal{L}_{J}\left(V^{C}(\Gamma, t: F)=1 \Rightarrow V^{C}(\Delta, t: F)=1\right)\right\}
\end{aligned}
$$

(f) for all $(c, A) \in \mathrm{CS}$ and for all $\Gamma \in W_{0}^{C}: E^{C}(\Gamma, c) \subseteq[A]$ and

$$
E(\Gamma, \underbrace{! \ldots !}_{n} c) \subseteq[\underbrace{! \ldots !}_{n-1} c: \ldots . . ! c: c: A] \text { for all } n \in \mathbb{N} .
$$

So the proofs are here:

(1) Since the empty set is proven to be $L_{C S}^{\star}$-consistent (see Lemma 9) it can be extended by the Lindenbaum Lemma to a maximal $\mathrm{L}_{\mathrm{CS}}^{\star}$-consistent set of formulas $\Gamma$ with $\Gamma \in W_{0}^{C}$. 
(2) Suppose $\Gamma \in W_{0}^{C}$ :

(a) We claim $V^{C}(\Gamma, \perp)=0$ : Suppose the opposite, then $V^{C}(\Gamma, \perp)=1$ hence by the definition of $V^{C}$ follows that $\perp \in \Gamma$. But this is a contradiction to the fact that $\Gamma$ is consistent.

(b) From left to right: Suppose $V^{C}(\Gamma, F \rightarrow G)=1$, then by the definition of $V^{C}, F \rightarrow G \in \Gamma$. Since $\Gamma$ is maximal $\mathrm{L}_{C S}^{\star}$-consistent this implies by Lemma 11 (3) that $F \notin \Gamma$ or $G \in \Gamma$. Hence again by the definition of $V^{C}, V^{C}(\Gamma, F)=0$ or $V^{C}(\Gamma, G)=1$.

From right to left: Suppose $V^{C}(\Gamma, F)=0$ or $V^{C}(\Gamma, G)=1$, then by the definition of $V^{C}$ either $F \notin \Gamma$ or $G \in \Gamma$. Since $\Gamma \in W_{0}^{C}, \Gamma$ is maximal $\mathrm{L}^{\star}$-consistent and hence in both cases by Lemma 11 (3) $F \rightarrow G \in \Gamma$. But this means again by the definition of $V^{C}$ that $V(\Gamma, F \rightarrow G)=1$.

(c) From left to right: Suppose $V^{C}(\Gamma, t: F)=1$, then by Definition 12 $t: F \in \Gamma$. Hence with the definition of $\Gamma / t$ we obtain $F \in \Gamma / t$. So for each $\Delta \in E^{C}(\Gamma, t), F \in \Delta$ (again by Definition 12). Hence for these $\Delta$ it follows by the definition of $V^{C}$ that $V^{C}(\Delta, F)=1$ and therefore $\Delta \in[F]$. Since this is true for all $\Delta \in E^{C}(\Gamma, t)$ we obtain $E^{C}(\Gamma, t) \subseteq[F]$. From right to left: The proof is by contraposition.

Suppose $V^{C}(\Gamma, t: F) \neq 1$, then by the definition of $V^{C} t: F \notin \Gamma$. We define a world $\Delta$ by $\Delta:=\Gamma / t$. Since $\Delta \in \mathcal{P}\left(\mathcal{L}_{J}\right)$ we can be sure that $\Delta$ exists, i.e. $\Delta \in W$. Since $t: F \notin \Gamma$ it follows that $F \notin \Gamma / t$ and therefore $F \notin \Delta$. But obviously $\Delta \supseteq \Gamma / t$ hence $\Delta \in E^{C}(\Gamma, t)$. So we conclude $E^{C}(\Gamma, t) \nsubseteq[F]$.

It remains to show that in case of $t=c^{\star}, \Delta:=\Gamma / t \in W_{M P}^{C}$ since otherwise $\Delta \notin E^{C}\left(\Gamma, \mathrm{c}^{\star}\right)$. In fact this is the case. Since $\Gamma \in W_{0}^{C}$ we obtain that $\Gamma$ is a maximal $\mathrm{L}_{\mathrm{CS}^{\star}}^{\star}$-consistent set of formulas and hence, whenever $\mathrm{c}^{\star}: A, \mathrm{c}^{\star}:(A \rightarrow B) \in \Gamma$ then by $\mathbf{j c}^{\star}$ we obtain $\mathrm{c}^{\star}: B \in \Gamma$. This means that whenever $A \in \Delta$ and $A \rightarrow B \in \Delta$ then $B \in \Delta$. Hence $\Delta=\Gamma / \mathrm{c}^{\star}$ is closed under modus ponens and therefore $\Delta \in W_{M P}^{C}$. So together with the former reasoning $\Delta \in E\left(\Gamma, \mathrm{c}^{\star}\right)$.

(3) Suppose $\Gamma \in W_{0}^{C}$ :

(a) Given some $F \in \mathcal{L}_{J}, s, t \in \mathrm{Tm}$ : We start by an observation on the relation between the sets $\Gamma /(s+t)$ and $\Gamma / s$ for $\Gamma \in W_{0}^{C}$. If $s: A \in \Gamma$ then since $\Gamma$ is maximal $\mathrm{L}_{\mathrm{CS}}^{\star}$-consistent $s+t: A \in \Gamma$ and therefore $\Gamma / s \subseteq \Gamma /(s+t)$. With the same reasoning $\Gamma / t \subseteq \Gamma /(s+t)$. Therefore if $\Delta \supseteq \Gamma /(s+t)$ then $\Delta \supseteq \Gamma / s$ and $\Delta \supseteq \Gamma / t$. This means that $E^{C}(\Gamma, s+t) \subseteq E^{C}(\Gamma, s)$ and $E^{C}(\Gamma, s+t) \subseteq E^{C}(\Gamma, t)$ and therefore $E^{C}(\Gamma, s+t) \subseteq E^{C}(\Gamma, s) \cap E^{C}(\Gamma, t)$.

(b) This follows directly from the definition of $E^{C}\left(\Gamma, \mathrm{c}^{\star}\right)$.

(c) If $\mathbf{j d}$ in $\mathrm{L}^{\star}$, then for any $\Gamma \in W_{0}^{C}$ we obtain $\neg(t: \perp) \in \Gamma$. Hence $\perp \notin \Gamma / t$. Therefore $\Gamma / t$ is $\mathrm{L}_{\mathrm{CS}}^{\star}$-consistent and can be expanded by the Lindenbaum Lemma to a maximal $\mathrm{L}_{\mathrm{CS}}^{\star}$-consistent set $\Delta \supseteq \Gamma / t$ with $\Delta \in W_{0}^{C}$ and $\Delta \in E^{C}(\Gamma, t)$.

(d) Assume for some $F \in \mathcal{L}_{J}, \Gamma \in W_{0}^{C}, t \in \mathrm{Tm}$ that $F \in \Gamma / t$, i.e. $t: F \in \Gamma$, since $\Gamma$ is maximal $\mathrm{L}_{C S}^{\star}$-consistent and $t: F \rightarrow F$ is an instance of the jt-axiom, we conclude that $F \in \Gamma$. Since $F$ was arbitrary we obtain $\Gamma \supseteq \Gamma / t$ and hence $\Gamma \in E^{C}(\Gamma, t)$. 
(e) Suppose for some $\Delta \in E^{C}(\Gamma, ! t)$, hence $\Delta \supseteq \Gamma / ! t$. Then assume for some arbitrary $F \in \mathcal{L}_{J}, V(\Gamma, t: F)=1$ i.e. by Definition $12 t: F \in \Gamma$. Since $\Gamma$ is maximal $\mathrm{L}_{\mathrm{CS}}^{\star}-$ consistent and $t: F \rightarrow ! t:(t: F)$ is an instance of the j4-axiom we obtain $! t:(t: F) \in \Gamma$ and hence $t: F \in \Gamma / ! t$. But then $t: F \in \Delta$ and by Definition 12 it follows that $V^{C}(\Delta, t: F)=1$. Since $F$ was an arbitrary formula and $\Delta$ an arbitrary world of $E^{C}(\Gamma, ! t)$ we conclude that the condition holds.

(f) Suppose $(c, A) \in \mathrm{CS}$, maximal $\mathrm{L}_{\mathrm{CS}}^{\star}$-consistency implies for all $\Gamma \in W_{0}^{C}$ that $c: A \in \Gamma$. Hence $A \in \Gamma / c$ and for all $\Delta \in E^{C}(\Gamma, c)$ we obtain $A \in \Delta$ and therefore $E^{C}(\Gamma, c) \subseteq[A]$.

Furthermore maximal $\mathrm{L}_{\mathrm{CS}}^{\star}$-consistency implies for all $\Gamma \in W_{0}$ by axiom necessitation that

$$
\underbrace{! \ldots !}_{n} c: \ldots: ! c: c: A \in \Gamma
$$

. Hence

$$
\underbrace{! \ldots !}_{n-1} c: \ldots: ! c: c: A \in \Gamma / \underbrace{! \ldots !}_{n} c
$$

and for all $\Delta \in E^{C}(\Gamma, \underbrace{! \ldots !}_{n} c)$ we obtain

$$
\underbrace{! \ldots !}_{n-1} c: \ldots: ! c: c: A \in \Delta
$$

and therefore

$$
E^{C}(\Gamma, \underbrace{! \ldots !}_{n} c) \subseteq[\underbrace{! \ldots ! !}_{n-1} c: \ldots: ! c: c: A]
$$

\section{The canonical model defined in Definition 21 is an

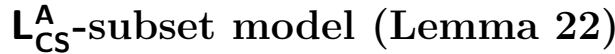

Proof. In order to prove that, we have to proceed in the same way as in the previous section, i.e. showing that $\mathcal{M}^{C}$ meets all the conditions we made for the valuation and the evidence function as well as the constant specification.

Since the canonical model is defined in the same way as the one of $\mathrm{L}_{\mathrm{CS}}^{\star}$-subset models, the corresponding proofs can be reused (see Lemma 13). Nevertheless, there is some difference. Instead of showing that $E^{C}\left(\Gamma, \mathrm{c}^{\star}\right) \subseteq W_{M P}^{C}$ we have to show that $E^{C}(\Gamma, s \cdot t) \subseteq\left\{\Delta \in W^{C} \mid \forall F \in \operatorname{APP}_{\Gamma}(s, t)(\Delta \in[F])\right\}$. Assume that we are given $\Gamma \in W_{0}^{C}, F \in \mathcal{L}_{J}^{\mathrm{A}}, s, t \in \mathrm{Tm}^{\mathrm{A}}$. Take any $\Delta \in E^{C}(\Gamma, s \cdot t)$, i.e. $\Delta \supseteq \Gamma /(s \cdot t)$. Hence for all $F$ s.t. $s \cdot t: F \in \Gamma$ we know that $F \in \Delta$. Hence by the definition of $V^{C}$, we have $V(\Delta, F)=1$ and therefore $\Delta \in[F]$.

It remains to show: if $F \in \operatorname{APP}_{\Gamma}(s, t)$ then $s \cdot t: F \in \Gamma$. Suppose for some formula $F$ that $F \in \operatorname{APP}_{\Gamma}(s, t)$ then by definition of $\operatorname{APP}_{\Gamma}(s, t)$ we know that there is a formula $H$ s.t. $E^{C}(\Gamma, s) \subseteq[H \rightarrow F]$ and $E^{C}(\Gamma, t) \subseteq[H]$. By 
using Lemma 22 (the part that corresponds to Lemma 13 (2c)) we conclude $V^{C}(\Gamma, s:(H \rightarrow F))=1$ and $V^{C}(\Gamma, t: H)=1$. Hence by the definition of $V^{C}$ we obtain $s:(H \rightarrow F) \in \Gamma$ and $t: H \in \Gamma$ and since $\Gamma$ is maximal $\mathrm{L}_{\mathrm{CS}^{\mathrm{A}}}$-consistent and $s:(H \rightarrow F) \rightarrow(t: H \rightarrow s \cdot t: F)$ is an instance of the $\mathbf{j}$-axiom we conclude that $s \cdot t: F \in \Gamma$.

\section{Soundness of PE-addapted $L^{\star}$-subset models (Theorem 27)}

PE-adapted $\mathrm{L}_{\mathrm{CS}}^{\star}$-subset models $\mathcal{M}$ are sound with respect to probabilistic evidence $\mathrm{PE}$, i.e. for all $F \in \mathrm{L}_{\text {prob }}$

$\mathrm{PE} \vdash F \Rightarrow \mathcal{M}, \omega \Vdash F$ for all PE-adapted $\mathrm{L}_{\mathrm{CS}}^{\star}$-subset models and all $\omega \in W_{0}$.

Proof. The proof is by induction on the length of the derivation of $F$ :

- If $F$ is derived by axiom necessitation or modus ponens or is an instance of axiom (1), then the proof is the analogue as in Theorem 3 since the relevant definitions have remained the same.

- If $F$ is an instance of axiom (2) the proof is analogue to the proof of Lemma 5: Suppose $\mathcal{M}, \omega \Vdash s:(A \rightarrow B)$ and $\mathcal{M}, \omega \Vdash t: A$ then $E(\omega, s) \subseteq[A \rightarrow B]$ and $E(\omega, t) \subseteq[A]$.

$$
\begin{aligned}
& E(\omega, s t)=E\left(\omega, s+t+c^{\star}\right) \subseteq \\
& E(\omega, s) \cap E(\omega, t) \cap E\left(\omega, c^{\star}\right) \subseteq[A \rightarrow B] \cap[A] \cap E\left(\omega, c^{\star}\right) .
\end{aligned}
$$

Hence for all $v \in E(\omega, s t)$ we have $V(v, A \rightarrow B)=1$ and $V(v, A)=1$ and $v \in E\left(\omega, c^{\star}\right)$ and therefore $V(v, B)=1$. Hence $E(\omega, s t) \subseteq[B]$ and we obtain $\mathcal{M}, \omega \Vdash s t: B$.

- If $F$ is an instance of axiom (3) then $F=(s: A \wedge t: A) \rightarrow[s \cup t: A]$ for some $A \in \mathrm{L}_{\text {prob }}, s, t \in \operatorname{Tm}^{\mathrm{P}}$. Suppose $\mathcal{M}, \omega \Vdash s: A \wedge t: A$ hence $E(\omega, s) \subseteq[A]$ and $E(\omega, t) \subseteq[A]$. Therefore $E(\omega, s \cup t) \subseteq E(\omega, s) \cup E(\omega, t) \subseteq[A]$ and since $\omega \in W_{0}$ we obtain $\mathcal{M}, \omega \Vdash s \cup t: A$.

- If $F$ is an instance of axiom (4) then either $F=1: A$ for some axiom $A$ or $0: G$ for some formula $G$.

Suppose $F=1: A$ for some axiom $A$. We assume that $\mathcal{M}, \omega \Vdash A$ for all $\omega \in W_{0}$, hence $E(\omega, 1)=W_{0} \subseteq[A]$ and therefore $\mathcal{M}, \omega \Vdash 1: A$ for all $\omega \in W_{0}$.

Suppose $F=0: G$ : For any $\omega \in W_{0}$ we have $E(\omega, 0)=\emptyset$ by Definition 26 . Since $\emptyset$ is a subset of any subset of $W$, we obtain $E(\omega, 0)=\emptyset \subseteq[G]$ for any formula $G \in \mathrm{L}_{\text {prob. }}$.

$-F$ is an instance of axiom (5). Assume $\mathcal{M}, \omega \Vdash t: X$ for some term $t$ and some formula $X$ and let $s \preceq t$. By (2) we find $t=s \cup t$. Thus

$$
E(\omega, t)=E(\omega, s \cup t)=E(\omega, s) \cup E(\omega, t)
$$

and therefore $E(\omega, s) \subseteq E(\omega, t)$. The assumption $\mathcal{M}, \omega \Vdash t: X$ means that $E(\omega, t) \subseteq[X]$. Hence we also get $E(\omega, s) \subseteq[X]$ and conclude $\mathcal{M}, \omega \Vdash s: X$. 


\section{References}

1. S. Artemov. On aggregating probabilistic evidence. In S. Artemov and A. Nerode, editors, LFCS 2016, pages 27-42. Springer, 2016.

2. S. Artemov and M. Fitting. Justification Logic: Reasoning with Reasons. Cambridge University Press, in preparation.

3. S. N. Artemov. Operational modal logic. Technical Report MSI 95-29, Cornell University, December 1995.

4. S. N. Artemov. Explicit provability and constructive semantics. Bulletin of Symbolic Logic, 7(1):1-36, March 2001.

5. S. N. Artemov. Justified common knowledge. TCS, 357(1-3):4-22, July 2006.

6. S. N. Artemov. The logic of justification. RSL, 1(4):477-513, Dec. 2008.

7. S. N. Artemov. The ontology of justifications in the logical setting. Studia Logica, 100(1-2):17-30, Apr. 2012.

8. S. N. Artemov and M. Fitting. Justification logic. In E. N. Zalta, editor, The Stanford Encyclopedia of Philosophy. Fall 2012 edition, 2012.

9. S. N. Artemov and E. Nogina. Topological semantics of justification logic. In E. A. Hirsch, A. A. Razborov, A. Semenov, and A. Slissenko, editors, CSR 2008,, pages 30-39. Springer, 2008.

10. A. Baltag, N. Bezhanishvili, A. Özgün, and S. Smets. Justified belief and the topology of evidence. In WoLLIC, 2016.

11. S. Bucheli, R. Kuznets, and T. Studer. Justifications for common knowledge. Applied Non-Classical Logics, 21(1):35-60, Jan.-Mar. 2011.

12. S. Bucheli, R. Kuznets, and T. Studer. Realizing public announcements by justifications. Journal of Computer and System Sciences, 80(6):1046-1066, 2014.

13. M. Fitting. The logic of proofs, semantically. APAL, 132(1):1-25, Feb. 2005.

14. R. Kuznets and T. Studer. Justifications, ontology, and conservativity. In T. Bolander, T. Braüner, S. Ghilardi, and L. Moss, editors, Advances in Modal Logic, Volume 9, pages 437-458. College Publications, 2012.

15. R. Kuznets and T. Studer. Weak arithmetical interpretations for the logic of proofs. Logic Journal of IGPL, 24(3):424-440, 2016.

16. R. Kuznets and T. Studer. Logics of Proofs and Justifications. College Publications, in preparation.

17. E. Lehmann and T. Studer. Subset models for justification logic. E-print 1902.02707, arXiv.org, 2019.

18. A. Mkrtychev. Models for the logic of proofs. In S. Adian and A. Nerode, editors, Logical Foundations of Computer Science, 4th International Symposium, LFCS'97, Yaroslavl, Russia, July 6-12, 1997, Proceedings, volume 1234 of LNCS, pages 266275. Springer, 1997.

19. V. Rantala. Impossible worlds semantics and logical omniscience. Acta Philosophica Fennica, 35:106-115, 1982. cited By 35.

20. V. Rantala. Quantified modal logic: Non-normal worlds and propositional attitudes. Studia Logica, 41(1):41-65, 1982.

21. J. van Benthem, D. F. Duque, and E. Pacuit. Evidence logic: A new look at neighborhood structures. In Advances in Modal Logic, 2012.

22. J. van Benthem, D. F. Duque, and E. Pacuit. Evidence and plausibility in neighborhood structures. CoRR, abs/1307.1277, 2014. 